\title{
Evaluating mental health services. A world perspective
}

\author{
Norman Sartorius
}

\section{Introduction}

Evaluations of mental health services are much in demand. Their results are supposed to help in improving the quality of mental health care and in making them economically better viable.

Yet, world-wide there is:

1) little agreement about the content of terms such as evaluation, mental health service, outcome of an activity although these and other terms are widely used;

2) uncertainty about the best use of results of evaluative research;

3) lack of consensus about who should evaluate what and by what method.

\section{Agreement on the definitions of terms used in evaluative research}

Evaluation is a term that has different meanings. In its most classical form evaluation denotes a comparison between results and goals of activity: in most instances the term is used to describe a process in which comparisons are difficult or impossible because

1) the results of the activity are described in terms which do not allow a comparison with the goals of the activity;

2) the goals of the activity are not defined in operational terms;

3) the period covered by the evaluation does not correspond to the period supposedly or explicitly covered by the goals;

4) confounding factors have not been considered at the point when the goals were set which makes the results of the evaluation invalid or difficult to interpret. 


\section{Areas of evaluation}

Measurements in the process of evaluation could be directed to

- the input (e.g. investment in terms of staff and buildings);

- the process (e.g. patients waiting time);

- the output (e.g. how much was done);

- the outcome of the activity (e.g. how many of these treated left the institution in a good state);

- the impact (e.g. on the image of the ministry of health).

The measurements of input and process are currently well established and used almost everywhere. What the terms cover, however, is defined differently in different studies. There is little agreement about the definition of personnel investment: so, for example, the services of an Indian psychiatrist in the UK are expressed in terms of his or her salary: the cost of $1 / 2$ producing + the psychiatrist, i.e. the cost of his education and upbringing in a different country could also be added to that investment line. In recent history such a definition of input investment was reflected in the demand by USSR authorities releasing graduate emigrants to Israel: the government made the granting of an emigration permit dependent on receiving a cash payment for the education which was given by the State to the emigrating specialist. Similar views, less explicit, were expressed by many who felt that an ethical relationship, between poor countries (producing university graduates and specialists) and rich countries (using them) should be based on the premise that the country receiving graduates must pay back the investment of the poor country by helping them to develop research and services.

Somewhat different theoretical considerations hold for measuring $1 / 2$ input + into buildings and similar facilities. The instauration of a mental health service - or a home for the mentally retarded, for example - often requires a considerable amount of work to convince the population (and particularly the immediate neighbours) that they should accept the service in their midst. Fear of the mentally ill and the stigma usually attached to institutions providing care for the mentally ill are profound and widespread; dealing with them in an appropriate manner is expensive. In many instances the authorities do not bother to send out teams and mount a public education campaign prior to opening a service: the cost in such cases - e.g. terms of anguish of the population and disagreeable incidents for staff and patients - is difficult to measure and is usually neglected. The same is true of other $1 / 2$ intangibles,+ e.g. the inconvenience and expense that may have to be incurred by patients because the new service is not near public transportation or was - in order to get a lower price - located far away from patients homes. These items are rarely mentioned in calculating cost of establishing a community mental health centre and yet have an important impact on the service.

Governments worldwide prefer to express the results of their activities in the field of mental health in terms of input: they state how many people receive a salary in the health system; they specify their professional qualifications and group staff in this manner although functionally they might have had to be grouped differently. Numbers of personnel by main activity which they do are rarely found in official statistics. Nurses on a ward may well spend half or more of their time looking after supplies, filling in 
forms or sitting in meetings; on official statistics they appear as having given $100 \%$ of their time to patient care.

The flow of activities and other elements of the process of care are measured in some settings but these measurements are often of doubtful validity. Even less frequently is it possible to find data about the proportions of staff who have (or have not) performed activities in accordance with some clearly written service guidelines. The time budget of staff in institutions is occasionally measured; the time budget of other participants in the care process (e.g. patient, family, neighbours) is occasionally assessed in special investigations but not routinely recorded (nor even asked about).

The invisible gains (and losses) of different types of services are usually known to those in services but not spoken about nor recorded. When a farmer's wife in Africa comes for a check-up or brings a child for a vaccination she may spend the morning waiting to see the nurse. The morning however might well be both agreeable and useful, not wasted as those working with middle-class urban populations might think. The time spent in talking with others might have been the first rest from a heavy daily routine in a long time and an occasion to learn from others who are also there, waiting, about health, disease, politics and family affairs. Other characteristics of the process of care - e.g. whether patients see the same doctor or nurse every time they come, how much they have understood from all that doctors told them and similar facts are rarely recorded although they could be used to improve service delivery. It is the process of care which will determine the satisfaction of the patients, the community and of staff with services given; yet facts relevant for the evaluation of satisfaction with service are rarely measured in a regular manner.

While evaluations of $1 / 2$ output + are reported from a number of settings in the developed world, it is usually difficult to interpret the data provided A statement that five thousand patients were treated in a hospital says little: did they have to be treated in hospital? have they been treated with respect? has their hospital stay speeded up or delayed their recovery? have they been provided a follow-up plan? have they lost their job or been marked for ever by their internment in the facility? Questions of this type remain without a reply and it is difficult to see how output data usually recorded and published could be used without additional information that can often be obtained only through ethnographic or other studies carried out in a regular manner in order to provide the contextual data necessary for the interpretation of output figures.

Recent years have seen several setbacks in the effort to use routine statistics in order to monitor or evaluate services. Many countries have decentralized the authority for mental health care to countries or districts and the center no longer receives nor stores data about the function of services. Local preferences will undoubtedly soon create so significant differences between the forms used for data collection and the time points when data are collected that the pooling of data and their comparison will become impossible. All the more important then - so it would seem - to carry out evaluative studies on service functioning: but, with the decentralization of services moneys needed for research are more difficult to obtain because, traditionally, provincial authorities did not fund research. Also, there are areas or provinces in which there 
are few scientists interested in applying for research funds even when these are made available.

Registers of psychiatric patients could have been tools to acquire and digest data about services: their usefulness has however been diminished in recent years because of financial difficulties, leading to staff reductions, human rights movements arguing against any systematic collection of data about individuals and the change of ethos in medicine with an emphasis on curative and private care models and a diminution of funds for public health service.

The outcome and impact of psychiatric care is also measured rarely and mainly in specific studies. Studies in this area are difficult to design and carry out; the interpretation of results often require considerable sophistication and close collaboration with many other disciplines inside and outside medicine. The time for a systematic evaluation of outcome and impact has clearly come: the development of technology for such studies and of sources of regular funding are becoming highly desirable for evaluative programmes.

\section{Definition of goals and needs for service evaluation}

Goal setting in programmes of care is also a technical component of evaluation that has to be developed and taught. An examination of service plans shows that goals and objectives are usually poorly formulated and cannot serve for comparison in evaluative programmes. Surrogates for evaluative models in which results are compared with results of similar programmes can be useful but cannot replace the evaluation that is based on well formulated objectives specific to the service.

It is possible to argue that goals of a programme should be stated in terms of satisfaction of needs. This is an interesting and useful way to proceed; the question which however arises is that of need definition. Previously needs were usually defined in medical terms. The total number of people with a given disease was taken as the measure of the need for service. This way of defining needs is losing popularity. A diagnosis rarely reflects different degrees of severity and does not incorporate facts such as the social class or social network of the person who is sick.

As years went by the wisdom of equating needs with disease prevalence begun to be questioned. The demands of the population - patients and their families - that were always considered as being of little validity and under the influence of the media and fashion gradually gained acceptance as a parameter for programme planning. The position that needs can be defined as the demands of the population for care to which the service can respond using means of demonstrated effectiveness now appear as being both rational and realistic.

If the trend of defining needs in this way gains strength, major changes in health service planning are bound to follow. What the population wants for those of its members who are suffering from dementia, for example, is the control of symptoms such as aimless wandering, screaming, undressing in public and incontinence. If this is accepted as the definition of needs on which planning for services will be based, the 
provision of cheap pampers and other ways of helping families who keep patients at home (e.g. by instruction, money and nursing personnel) will be the right response rather than the building of a residential or psychiatric service with highly qualified staff members which would be entrusted with the (medical) care of such patients. Since the definition of needs in this way is very dependent on the sociocultural setting in which care is to take place, the same disease may lead to different statements of needs and the fact that we know how many cases of a disease there are in a population will be valuable only if we also know regional variations of such numbers and have information about the expectations of the population, its ways of living and dealing with them and its areas of intolerance. Such data are only gradually becoming available from focused studies and we are still far from the introduction of questions about these matters into ordinary medical records and statistics.

The above example also illustrates the difficulties in defining mental health services. Does the provision of pampers at a reduced rate to families who are looking after a demented person represent rendering mental health service? Are the words $1 / 2$ mental health service + to be reserved only for acts which personnel qualified in psychiatry performs to help patients with mental disorders? Or should the term mental health service be reserved for the description of institutions dealing with mental disorder? And if we plan services, should we plan for institutions or the number of units of care to be provided to a well defined population by a variety of people and institutions?

\section{Uncertainty about the desirability of evaluation}

Evaluation often meets with considerable active and passive resistance. There are several reasons that at least partly contribute to this negative attitude: first, evaluation has, not infrequently, been used to remove persons rather than to advance a programme. Also, it often precedes a reform of services which is not welcome to staff. Second, results of evaluation are often used to justify a decision that was already made; third, evaluation is often costly. Loose resources that could be used to carry out evaluation do not abound: consequently, resources for evaluative work have to be taken from other programmes already underway. Fourth, the analysis of results of evaluation often takes so much time that results cannot be used for any practical purposes. Fifth, objectives of programmes are often formulated in a manner that corresponds to a given situation at the point of starting the programme. New scientific findings or changes in sociocultural factors may have made the programme take a new course: the objectives however are only rarely examined and reformulated in a systematic manner so that at the point of evaluation the original objectives are of no use.

Sixth, the indicators which are used in evaluative work are often selected without much thought about the impact that measuring one and not another matter may have on the image of the service and of psychiatry. Concentration, for example, on statistics about assaults by the mentally ill supports the notion that the mentally ill are violent; counting side effects of treatment confirms the prejudice that psychiatric treat- 
ment is not only cruel but also dangerous; reporting the numbers of $1 / 2$ escapes + from services reinforces the image of the mental hospital as a prison.

\section{The evaluators}

It is not easy to decide who should be entrusted with the evaluation of a mental health programme. Previously it was usual that the government authority which has established the service carries out the evaluation. This manner of proceeding came under considerable criticism because of the possibility that poor quality of service will not be reported because it might tarnish the administration's image. The fact that the abuse of psychiatry for political purposes could continue for a long time in government owned institutions was also an argument that was bound to lead to a change in the evaluatory system. Several options were proposed: self-evaluation by staff; evaluation through the eyes of the population by exploring in a regular manner what they felt about the service (e.g. in Cuba); evaluation by a special agency within the ministry of health run independently from the hierarchy of officers organizing the psychiatric care (similar to the Dutch system); hiring of independent consultant firms; evaluation by the psychiatric society of the country. Each of these models has advantages and disadvantages. Some of these are listed below:

Evaluator

Staff themselves

Government agency or officials

Non-governmental organization-

Population
Adavantage

cheap can be made part of daily service

temporal stability link to funding authorities

involves professionals who know the recent scientific facts

provides a fresh and important view which may not coincide with the views of the professional organizations or the government
Disadvantage

can be biased

staff often reluctant to cooperate

may be overemphasizing matters of interest to the profession

complex to organize 
Experience from different countries seems to indicate that the most useful evaluation might have the following characteristics:

- there should be several sources of data for the evaluation including at least staff themselves, the population served and an independent observer;

- results of evaluation should be primarily used for the improvement of the service in which data was obtained; secondary uses of data might include scientific publications, government reports, and the production of training materials;

- results of evaluational exercises are an input into the process of planning for the future but should not determine the nature and shape of these plans: the fact that nobody seems to object to long in-patient treatment does not mean that a shorter inpatient stay might not have achieved as much and that we should not strive for it in the future;

- the cost of evaluation must be foreseen in plans of services. These should also include funds for the training of staff in evaluational technology.

\section{Conclusions}

Evaluation of mental health care is a tool that can help in reaching rational decisions about the way in which mental health services should be organized and performed. To be able to serve in this way, evaluation must be seen as one of the inputs into decision making rather than as the determinant of decisions; it should be based on information from several sources including staff, the population served and independent observers; and it should be incorporated into the functioning of the services in a manner that will save staff time and avoid interference with the provision of care.

Among future tasks aiming to optimize evaluation, two loom particularly large: (i) to reach an agreement on terminology and methods of evaluation; and (ii) to educate those concerned about the way in which evaluational data should be obtained and used. Professional societies could play an important role in respect of both tasks and should be given the responsibility and opportunity to make their contribution. 